\title{
Condição periodontal de crianças e adolescentes com diabetes melito tipo 1
}

\author{
Periodontal status in children and adolescents with type 1 diabetes mellitus
}

Andréa Cristina Vilan Xavier', Ivani Novato Silva', Fernando de Oliveira Costa ${ }^{2}$, Daniela Soares Corrêa ${ }^{3}$

\begin{abstract}
RESUMO
Objetivo: Avaliar a condição periodontal de crianças e adolescentes diabéticos e fatores relacionados. Métodlos: $\mathrm{O}$ índice de placa (IPL), sítios com sangramento à sondagem (SS), profundidade de sondagem (PS) e nível de inserção clínica (NIC) foram avaliados em todos os dentes permanentes em oclusão de 168 diabéticos tipo 1, não fumantes, com $13 \pm 3,5$ anos de idade. IPL e SS foram avaliados também em dentes decíduos. Resultados: Observou-se prevalência de 20,8\% de gengivite e $5,9 \%$ de periodontite. Indivíduos com mau controle metabólico tiveram maiores percentuais de sítios com alteração da PS $(p=0,004)$ e NIC $(p=0,014)$. Indivíduos com $>5$ anos de doença apresentaram maiores percentuais de sítios afetados à avaliação da PS $(p=0,002)$, NIC $(p=0,007)$ e SS $(p<0,001)$. Conclusões: Maior duração do diabetes melito tipo 1 e mau controle metabólico foram significativamente associados a alterações periodontais indicando maior suscetibilidade para doença periodontal nessa população. Arq Bras Endocrinol Metab. 2009;53(3):348-54.
\end{abstract}

Descritores

Adolescente; dentição; periodontite; diabetes melito

\begin{abstract}
Objective: To evaluate periodontal condition of diabetic children and its related factors. Methods: The plaque index (PI), sites with bleeding on probing (BOP), probing depth (PD) and clinical attachment level (CAL) were evaluated in all occlusion permanent teeth of 168 non smoking type 1 diabetic children, $13 \pm 3.5$ years old. The PI and BOP evaluations were also performed in deciduous teeth. Results: It was observed a prevalence of $20.8 \%$ of gingivitis and $5.9 \%$ of periodontitis. Those individuals with poor metabolic control had higher percentage of affected sites on PD ( $p=0.004)$ and on CAL $(p=0.014)$. Patients having more than five years with diabetes mellitus type 1 showed higher percentual of affected sites on $\operatorname{PD}(p=0.002)$, on BOP $(p<0.001)$ and on CAL $(p=0.007)$. Conclusions: DM1 duration and poor glycemic control were significantly associated with periodontal disturbances suggesting higher susceptibility of this population in developing DP. Arq Bras Endocrinol Metab. 2009;53(3):348-54.
\end{abstract}

Keywords

Adolescent; dentition; periodontitis; diabetes melito

Ivani Novato Silva

Divisão de Endocrinologia Dediátrica, Hospital das Clín

Faculdade de Medicina, UFMG

Av. Alfredo Balena, 190, sala 267

30130-100 - Belo Horizonte, MG

Brasil

ivanins@medicina.ufmg.br

Recebido em 17/Set/2008

Aceito em 12/Jan/2009
A doença periodontal (DP) é uma reação inflamatória infecciosa dos tecidos gengivais (gengivite) ou suporte dos dentes: ligamento periodontal, cemento e osso alveolar (periodontite) devido à ação de um grupo de bactérias específicas, que se manifestam provocando danos nos tecidos periodontais (1). Um dos principais fatores de risco sistêmico para a DP é diabetes melito
(DM), disfunção metabólica crônica caracterizada pela hiperglicemia resultante da deficiência da secreção ou ação da insulina (2,3-5).

O DM é associado à alta morbimortalidade e sua ocorrência vem aumentando. A Organização Mundial da Saúde (OMS) caracteriza o problema como epidemia global, que atinge mais de 245 milhões de pessoas 
no mundo (6). No Brasil, considerando-se os dados obtidos pelo Censo realizado em 1989 pelo Ministério da Saúde (MS) e pela Sociedade Brasileira de Diabetes (SBD), observou-se a prevalência de $7,6 \%$ na faixa etária de 30 a 69 anos (7). De todos os casos, $5 \%$ a $10 \%$ apresentam DM do tipo 1 (DMl), com pouca ou nenhuma capacidade de secreção de insulina.

A associação entre a DP e o DM está relacionada a vários fatores como suscetibilidade individual, idade, controle glicêmico, duração do diabetes, cuidados com a saúde bucal e hábitos como, por exemplo, o de fumar.

O desenvolvimento da DP resulta de um processo multifatorial. Para que ela ocorra, é necessário que haja um desequilíbrio entre os mecanismos protetores do organismo e a quantidade de bactérias periodontopatogênicas associado a fatores de risco. Em pacientes diabéticos ocorrem alterações vasculares, alterações imunológicas, como disfunção de leucócitos polimorfonucleares, e aumento da produção de mediadores inflamatórios e alterações metabólicas no tecido conjuntivo, que interferem no processo de cicatrização e podem afetar qualquer tecido, incluindo o periodonto, que justificam a maior probabilidade de esses indivíduos desenvolverem a DP (3,8-10). Defeitos na função neutrofílica, alterações na renovação do colágeno e no padrão normal de cicatrização são fatores também relacionados ao padrão de evolução da DP nos pacientes diabéticos. A produção aumentada de AGES (advanced glication endproducts), produtos finais da glicosilação e oxidação não enzimática das proteínas e lípides, parece ter importante papel no desenvolvimento dos distúrbios periodontais, juntamente com outros fatores como a hiperglicemia intracelular e os anteriormente citados (11).

Assim como o DM pode estar relacionado a alterações nos padrões de evolução da DP, sendo considerado fator de risco, a gravidade da DP tem sido associada também ao mau controle metabólico, numa relação bidirecional. Infecções periodontais podem, como qualquer outro tipo de infecção, dificultar o controle glicêmico do paciente diabético, predispondo-o à resistência insulínica $\mathrm{e}$ desencadeando um estado de hiperglicemia crônica (3). Tem-se sugerido que esse desequilíbrio do metabolismo glicêmico predispõe o paciente à inflamação gengival e ao maior risco de desenvolvimento da DP.

Em revisão da literatura, observou-se, no entanto, que a maioria dos estudos é conduzida em indivíduos adultos e com DM tipo 2; dessa forma, dados em indivíduos com DMl permanecem escassos e conflitantes. Por esse motivo, avaliamos a condição periodontal e a sua relação com o grau de controle metabólico e outras variáveis de interesse em crianças e adolescentes diabéticos tipo 1 .

\section{MÉTODOS}

A estratégia amostral foi determinada por recenseamento, isto é, foram examinados todos os indivíduos que frequentaram o Ambulatório de Endocrinologia Pediátrica do Hospital das Clínicas da Universidade Federal de Minas Gerais (HC-UFMG) no período de fevereiro de 2005 a novembro de 2006 em dias de semana alternados e aleatórios.

Assim, de um universo de 300 pacientes, foram examinados 168 crianças e adolescentes com DMl de ambos os gêneros, na faixa etária de 7 a 19 anos, atendidos na Divisão de Endocrinologia Pediátrica do HC-UFMG.

Foram excluídos indivíduos em tratamento ortodôntico, portadores de qualquer outra doença sistêmica ou síndrome associada à suscetibilidade à DP, fumantes e aqueles indivíduos com idade inferior a 7 e superior a 19 anos.

O estudo de delineamento transversal foi aprovado pelo Comitê de Ética em Pesquisa da UFMG.

Os indivíduos que preencheram os critérios de inclusão e concordaram em participar responderam a um questionário contendo informações sobre as variáveis de riscos sociais e comportamentais que pudessem interferir na gravidade da DP. Em seguida, foram submetidos a um criterioso exame clínico intrabucal realizado no próprio consultório médico do HC por um único examinador previamente calibrado, após consulta médica de rotina e em boas condições de iluminação. $\mathrm{O}$ instrumental utilizado no exame foi espelho clínico e sonda periodontal milimetrada (marca Trinity ${ }^{\circledR}$ ) e roletes de algodão esterilizados em autoclave. Todos os procedimentos foram realizados seguindo-se as normas de biossegurança; paramentação profissional: avental, gorro, máscara, óculos de proteção e luvas descartáveis.

A determinação clínica do estado periodontal foi feita por exame objetivo em todos os dentes permanentes em oclusão, com exceção dos terceiros molares, para avaliação dos seguintes parâmetros periodontais: índice de placa, sangramento à sondagem, profundidade de sondagem e o nível clínico de inserção.

$\mathrm{O}$ índice de placa (IPL) foi caracterizado pelos seguintes escores (12):

0 = ausência de placa; 1 = visualização da placa através de sua remoção por sonda (visível); 2 = placa visível 
clinicamente (moderada); 3 = placa abundante inclusive nos espaços interdentais (abundante).

Para a caracterização desse índice, a cavidade bucal é dividida em seis sextantes e atribui-se a cada um deles o escore correspondente ao elemento dental mais comprometido do sextante. A partir da somatória dos seis escores que representam a cavidade bucal, obtêm-se a porcentagem de placa bacteriana do indivíduo examinado e o índice de higiene bucal (IHB), assim caracterizado: se $<33 \%$, bom estado de higiene bucal; $\geq 34 \mathrm{e}$ $<66 \%$, mau estado de higiene e acima de $67 \%$ de placa bacteriana, péssimo estado de higiene bucal.

A profundidade de sondagem (PS) foi medida com sonda milimetrada, em todos os dentes permanentes em oclusão na cavidade bucal, com exceção dos terceiros molares, de forma circunferencial nas superfícies vestibular, lingual/palatina, mesial e distal. O registro na ficha clínica periodontal foi feito para valores a partir de $4 \mathrm{~mm}$ de forma ordinal em escala de $1 \mathrm{~mm}(2)$.

$\mathrm{O}$ sangramento à sondagem (SS) foi definido pela leitura clínica dos sítios sangrantes realizada no momento da medida da PS ou até 15 segundos após e analisado de forma dicotômica: presença $(+)$ ou ausência (-) de sangramento nas superfícies examinadas (mesial, distal, vestibular, lingual/palatina) $(13,14)$.

O nível clínico de inserção (NIC) foi medido com sonda milimetrada por meio da leitura do limite amelocementário ao fundo do sulco ou bolsa periodontal (superfícies vestibular e lingual/palatina). Foram registrados valores de NIC $\geq 3 \mathrm{~mm}$ de forma ordinal em escala de $1 \mathrm{~mm}$. Em dentes decíduos, realizaram-se apenas IPL e SS.

A DP foi classificada como: (A) gengivite, caracterizada pela presença de $25 \%$ ou mais de sítios com sangramento à sondagem e nenhum sítio com perda de inserção clínica $>2 \mathrm{~mm}$ (15), (B) periodontite, caracterizada pelo achado de pelo menos um sítio apresentando simultaneamente PS $\geq 4 \mathrm{~mm}$ e NIC $\geq 3 \mathrm{~mm}$ (2).

Teste de concordância Kappa interexaminador foi realizado para os parâmetros clínicos PS, NIC e SS revelando valores $\geq 0,75$.

Os dados coletados foram registrados em ficha clínica periodontal padronizada no curso de Especialização em Periodontia do Departamento de Odontologia da Pontifícia Universidade Católica de Minas Gerais (DO/ PUC-MG).

O controle glicêmico foi avaliado por meio da média dos últimos dois anos da hemoglobina glicada ( $\mathrm{HbAlc}$ ) realizada a cada três meses. As dosagens da HbAlc foram feitas no Laboratório Central do HC-UFMG pelo método HPLC (VR = 4\%-6,5\%). O grau de controle glicêmico foi caracterizado de acordo com a avaliação proposta por Chase, dividindo-se o valor do resultado da HbAlc do paciente pelo limite superior de normalidade do método. Considera-se bom controle uma relação de $\mathrm{HbAlC}<1,33$; controle regular se $\mathrm{HbAlC} \geq$ $1,33 \leq 1,49$ e mau controle se $\mathrm{HbAlC} \geq 1,5$ (16).

\section{Análise estatística}

Foi feita uma análise descritiva das variáveis independentes de interesse dividindo-se a amostra em indivíduos com dentição mista ou permanente.

Para análise, foram utilizadas as porcentagens de sítios sangrantes, porcentagens de sítios com PS $\geq 4 \mathrm{~mm}$, porcentagens de sítios com NIC $\geq 3 \mathrm{~mm}$ e a média do sextante mais comprometido pelo IHB.

Os testes qui-quadrado $\left(\chi^{2}\right)$ e Exato de Fisher (quando indicado) foram usados para avaliar as associações entre os grupos (dentição mista e permanente) em relação ao número diário de escovações, uso de fio dental, visitas ao dentista, IHB, HbAlc e critérios PS/ NIC, e para avaliar a influência do IHB, escovação, uso de fio dental e gênero no SS. O teste de Mann-Whitney foi aplicado para avaliar a influência do tipo de dentição em relação ao controle glicêmico, IHB e SS e a influência do tempo de diabetes dicotomizado em menor e maior ou igual a cinco anos nos parâmetros periodontais: PS, SS, IHB e NIC. As comparações entre o grau de controle glicêmico e as variáveis periodontais PS, SS, IHB e NIC foram realizadas utilizando-se o teste de Kruskal-Wallis. Todos os resultados foram considerados significativos para uma probabilidade de significância inferior a $5 \%(\mathrm{p}<0,05)$.

\section{RESULTADOS}

Participaram do estudo 168 indivíduos, sendo 74 (44\%) do gênero masculino. Os pacientes foram caracterizados segundo o tipo de dentição (Tabela 1$)$. Dos pacientes com dentição mista $(n=60), 26(43,3 \%)$ eram do gênero masculino. Entre os pacientes com dentição permanente $(\mathrm{n}=108), 48(44,4 \%)$ eram do gênero masculino. A idade variou de 7 a 19 com média de $13 \pm 3,5$ anos. Todos os pacientes eram não fumantes e $98,1 \%$ deles eram estudantes. Entre os pais/responsáveis, 58,3\% tinham até ${ }^{\circ}$ o grau completo e $81 \%$ das famílias apresentaram renda de até cinco salários mínimos, caracterizando uma amostra com predomínio de baixo poder aquisitivo. 
No momento da avaliação, os pacientes apresentaram tempo de diabetes (TD) de 0,4 a 18 anos com média de $6,1 \pm 3,7$.

O percentual de pacientes com dentição permanente que escovavam os dentes mais de três vezes ao dia e faziam uso diário do fio dental era significativamente maior que o número de pacientes com dentição mista ( $\mathrm{p}<0,001$ e $\mathrm{p}=0,003$, respectivamente). Não houve associação significativa entre o tipo de dentição e as variáveis: controle glicêmico, IPL e critérios PS $\geq 4 \mathrm{~mm}$ / NIC $\geq 3 \mathrm{~mm}$ associados com ocorrência simultânea em pelo menos um sítio (Tabela $\mathrm{l}$ ).

A prevalência de gengivite na população foi de $20,8 \%(\mathrm{n}=35)$. Destes, $22(62,8 \%)$ eram do gênero

\begin{tabular}{|c|c|c|c|c|c|}
\hline \multirow{3}{*}{ Variáveis } & \multicolumn{4}{|c|}{ Dentição } & \multirow[b]{3}{*}{ Total } \\
\hline & \multicolumn{2}{|c|}{ Mista } & \multicolumn{2}{|c|}{ Permanente } & \\
\hline & $\mathrm{n}$ & $\%$ & n & $\%$ & \\
\hline \multicolumn{6}{|l|}{ Gênero } \\
\hline Masculino & 26 & 43,3 & 48 & 44,4 & 74 \\
\hline Feminino & 34 & 56,7 & 60 & 55,6 & 94 \\
\hline \multicolumn{6}{|l|}{ Idade à avaliação } \\
\hline$<12$ anos & 55 & 91,7 & 16 & 14,8 & 71 \\
\hline$\geq 12$ anos & 5 & 8,3 & 92 & 85,2 & 97 \\
\hline \multicolumn{6}{|c|}{ Tempo de diabetes à avaliação } \\
\hline$<5$ anos & 35 & 44,8 & 43 & 55,2 & 78 \\
\hline$\geq 5$ anos & 25 & 27,7 & 65 & 72,3 & 90 \\
\hline \multicolumn{6}{|l|}{ Escovação } \\
\hline $1-2$ vezes & 33 & 55,0 & 28 & 25,9 & 66 \\
\hline$\geq 3$ vezes & 27 & 45,0 & 80 & 74,1 & 102 \\
\hline \multicolumn{6}{|c|}{ Uso de fio dental ${ }^{*}(p=0,003)$} \\
\hline Sim & 11 & 18,3 & 44 & 40,7 & 55 \\
\hline Não & 49 & 81,7 & 64 & 59,3 & 113 \\
\hline \multicolumn{6}{|c|}{ Índice de placa $(I P L)^{*}(p=0,059)$} \\
\hline Visível & 3 & 83,3 & 19 & 17,6 & 22 \\
\hline Moderada & 17 & 28,3 & 23 & 21,3 & 40 \\
\hline Abundante & 40 & 66,7 & 66 & 61,1 & 106 \\
\hline \multicolumn{6}{|c|}{ Controle glicêmico* $(p=0,43)$} \\
\hline$<1,33 \mathrm{HbA1c}$ & 16 & 26,7 & 27 & 25,0 & 43 \\
\hline $1,33 \geq 1,49 \mathrm{HbA} 1 \mathrm{c}$ & 18 & 30,0 & 24 & 22,2 & 42 \\
\hline$\geq 1,5 \mathrm{HbA1C}$ & 26 & 43,3 & 57 & 52,8 & 83 \\
\hline \multicolumn{6}{|c|}{$P S \geq 4$ e NIC $\geq 3 \mathrm{~mm}^{\star \star * \star *}(p=0,498)$} \\
\hline Não & 58 & 96,7 & 100 & 92,6 & 158 \\
\hline $\operatorname{Sim}$ & 2 & 3,3 & 8 & 7,4 & 10 \\
\hline
\end{tabular}

PS: profundidade de sondagem; NIC: nível clínico de inserção.

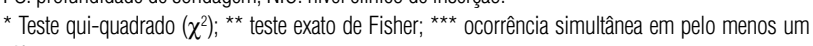
sítio. masculino e 20 (80\%) apresentaram controle glicêmico ruim. Dos 168 pacientes examinados, $88(52,3 \%)$ apresentaram pelo menos um sítio com sangramento à sondagem. Escovação e uso diário de fio dental não mostraram associação significativa com o percentual de sítios com sangramento à sondagem.

O percentual de sítios sangrantes (com gengivite) teve associação significativa com o IPL e higiene bucal. O percentual de pacientes com presença de sangramento à sondagem foi significativamente maior $(\mathrm{p}<0,001)$ entre aqueles com placa abundante (péssimo estado de higiene bucal) e o percentual de indivíduos com placa abundante foi significativamente maior no gênero masculino $(\mathrm{p}<0,001)$.

Dez pacientes (três do gênero masculino e oito com dentição permanente) apresentavam periodontite (presença de pelo menos um sítio com PS $\geq 4 \mathrm{~mm}$ e NIC $\geq$ $3 \mathrm{~mm}$ no mesmo sítio), o que corresponde a $5,9 \%$ da população estudada.

De acordo com a média das HbAlc, 49,4\% ( $\mathrm{n}=83$ ) dos pacientes tinham controle glicêmico ruim. Os pacientes com mau controle metabólico apresentaram mais alterações das variáveis periodontais: aqueles com índices maiores que 1,5 para $\mathrm{HbAlc}$ tiveram percentuais significativamente maiores de sítios afetados na PS e na NIC. Quando a amostra foi estratificada pelo tipo de dentição, as associações significativas persistiram somente em pacientes com dentição permanente (Tabela 2).

O tempo de diabetes também esteve significativamente associado com os parâmetros periodontais: maiores índices de PS $(\mathrm{p}=0,002)$, SS $(\mathrm{p}<0,001) \mathrm{e}$ NIC ( $\mathrm{p}=0,007)$ estavam presentes em indivíduos com cinco anos ou mais de doença. Quando a amostra foi estratificada pelo tipo de dentição, essas associações só apareceram também em pacientes com dentição permanente. A relação entre variáveis periodontais e tempo de diabetes é apresentada na tabela 3 .

\section{DISCUSSÃO}

As inúmeras alterações que ocorrem sistemicamente no indivíduo diabético o predispõem à ocorrência de distúrbios no periodonto. Em muitos estudos tem sido constatada associação entre gravidade e progressão da DP com o DM, além de sua prevalência maior (17-24). A heterogeneidade metodológica observada como diferenças nas populações estudadas, na avaliação dos parâmetros de controle glicêmico e periodontais e a própria caracterização da DP, no entanto, dificultam possíveis comparações e conclusões mais definitivas. 


\begin{tabular}{|c|c|c|c|c|c|c|c|c|c|}
\hline \multirow{3}{*}{ Variável } & \multicolumn{9}{|c|}{ Controle metabólico } \\
\hline & \multicolumn{3}{|c|}{ Bom $(n=43)$} & \multicolumn{3}{|c|}{ Regular $(n=42)$} & \multicolumn{3}{|c|}{ Ruim (n = 83) } \\
\hline & Mín. & Máx. & Mediana & Mín. & Máx. & Mediana & Mín. & Máx. & Mediana \\
\hline$P S(p=0,004)$ & 0,0 & 0,0 & 0,0 & 0,0 & 0,0 & 0,0 & 0,0 & 0,3 & 0,0 \\
\hline$S S(p=0,488)$ & 0,0 & 49,1 & 0,0 & 0,0 & 44,6 & 8,6 & 0,0 & 65,2 & 6,3 \\
\hline IHB $(p=0,308)$ & 1 & 3 & 3 & 1 & 3 & 3 & 1 & 3 & 3 \\
\hline NIC $(p=0,014)$ & 0,0 & 0,0 & 0,0 & 0,0 & 0,0 & 0,0 & 0,0 & 6,5 & 0,0 \\
\hline \multicolumn{10}{|c|}{ Pacientes com dentição mista $(n=60)$} \\
\hline$P S(p=0,27)$ & 0,0 & 0,0 & 0,0 & 0,0 & 0,0 & 0,0 & 0,0 & 0,1 & 0,0 \\
\hline SS $(p=0,58)$ & 0,0 & 27,1 & 2,1 & 0,0 & 39,6 & 9,8 & 0,0 & 52,1 & 0,0 \\
\hline $\operatorname{HB}(p=0,38)$ & 1 & 3 & 3 & 2 & 3 & 3 & 1 & 3 & 3 \\
\hline $\operatorname{NIC}(p=0,27)$ & 0,0 & 0,0 & 0,0 & 0,0 & 0,0 & 0,0 & 0,0 & 2,1 & 0,0 \\
\hline \multicolumn{10}{|c|}{ Pacientes com dentição permanente $(n=108)$} \\
\hline$P S(p=0,02)$ & 0,0 & 0,0 & 0,0 & 0,0 & 0,0 & 0,0 & 0,0 & 0,3 & 0,0 \\
\hline$S S(p=0,35)$ & 0,0 & 49,1 & 0,0 & 0,0 & 44,6 & 4,0 & 0,0 & 65,2 & 14,3 \\
\hline $\mathrm{HB}(\mathrm{p}=0,41)$ & 1 & 3 & 3 & 1 & 3 & 3 & 1 & 3 & 3 \\
\hline NIC $(p=0,06)$ & 0,0 & 0,0 & 0,0 & 0,0 & 0,0 & 0,0 & 0,0 & 6,5 & 0,0 \\
\hline
\end{tabular}

PS: \% sítios com PS $\geq 4$ mm; SS: \% sítios com sangramento à sondagem; IHB: escore determinado pela porcentagem do IPL; NIC: \% sítios com NIC $\geq 3 \mathrm{~mm}$.

${ }^{*}$ Teste de Kruskal-Wallis;

\begin{tabular}{|c|c|c|c|c|c|c|}
\hline \multirow{3}{*}{ Variável } & \multicolumn{6}{|c|}{ Tempo de diabetes (TD) } \\
\hline & \multicolumn{3}{|c|}{ TD $<5$ anos $(n=78)$} & \multicolumn{3}{|c|}{$\mathrm{TD} \geq 5$ anos $(\mathrm{n}=90)$} \\
\hline & Mín. & Máx. & Mediana & Mín. & Máx. & Mediana \\
\hline$P S(p=0,002)$ & 0,0 & 0,0 & 0,0 & 0,0 & 0,3 & 0,0 \\
\hline$S S(p<0,001)$ & 0,0 & 50,0 & 0,0 & 0,0 & 65,2 & 13,4 \\
\hline $\mathrm{IHB}(\mathrm{p}=0,844)$ & 1 & 3 & 3 & 1 & 3 & 3 \\
\hline $\mathrm{NIC}(p=0,007)$ & 0,0 & 0,0 & 0,0 & 0,0 & 6,5 & 0,0 \\
\hline \multicolumn{7}{|c|}{ Pacientes com dentição mista $(n=60)$} \\
\hline PS $(p=0,09)$ & 0,0 & 0,0 & 0,0 & 0,0 & 0,1 & 0,0 \\
\hline$S S(p=0,68)$ & 0,0 & 39,6 & 4,2 & 0,0 & 52,1 & 0,0 \\
\hline $\operatorname{IHB}(p=0,97)$ & 1 & 3 & 3 & 1 & 3 & 3 \\
\hline $\operatorname{NIC}(p=0,09)$ & 0,0 & 0,0 & 0,0 & 0,0 & 2,1 & 0,0 \\
\hline \multicolumn{7}{|c|}{ Pacientes com dentição permanente $(n=108)$} \\
\hline$P S(p=0,002)$ & 0,0 & 0,0 & 0,0 & 0,0 & 0,3 & 0,0 \\
\hline$S S(p<0,001)$ & 0,0 & 50,0 & 0,0 & 0,0 & 65,2 & 17,0 \\
\hline $\mathrm{IHB}(\mathrm{p}=0,844)$ & 1 & 3 & 3 & 1 & 3 & 3 \\
\hline $\operatorname{NIC}(p=0,041)$ & 0,0 & 0,0 & 0,0 & 0,0 & 6,5 & 0,0 \\
\hline
\end{tabular}

PS: \% sítios com PS $\geq 4$ mm; SS: \% sítios com sangramento à sondagem; IHB: escore determinado pela \% do IPL; NIC: \% sítios com NIC $\geq 3$ mm.

*Teste de Mann-Whitney.

Por outro lado, os critérios para a definição de caso e gravidade da DP também têm sido objeto de controvérsias. A maioria dos estudos epidemiológicos, conduzidos até o final da década de 1990, utilizou critérios para definição de periodontite com baixo ponto de corte para os parâmetros clínicos de PS e NIC (9). Entretanto, na literatura atual é consenso a caracterização de
PS $>3 \mathrm{~mm}$ como um aprofundamento patológico do sulco gengival, denominado bolsa periodontal (13). Na avaliação das crianças e adolescentes do presente trabalho, optou-se por considerar o ponto de corte para $\mathrm{PS} \geq 4 \mathrm{~mm}$ e para a mensuração padrão-ouro em periodontia, NI $\geq 3 \mathrm{~mm}$, com o objetivo de identificar todos os possíveis indivíduos com DP e eliminar falso- 
positivos. A combinação desses dois critérios tem sido considerada segura na identificação de indivíduos com periodontite (25).

O achado de dez crianças e adolescentes com periodontite, correspondente a $5,9 \%$ da população estuda$\mathrm{da}$, representa uma prevalência relativamente elevada se comparada a dados da literatura para pacientes saudáveis nessa faixa etária. Os dados de ocorrência de periodontites mais graves, crônica e agressiva em crianças e adolescentes saudáveis são muito discrepantes quando avaliados mundialmente. Em uma revisão de literatura, Albandar (26) descreveu prevalências em vários países variando de $0,1 \%$ a $20 \%$ e, na América do Sul, de $0,3 \%$ a $1,0 \%$ para a periodontite agressiva e 4,0 a $8,0 \%$ para a periodontite crônica em crianças e adolescentes. $\mathrm{Na}$ República Dominicana, Colins e cols. (27), estudando 2007 adolescentes, encontraram prevalência de 4,0\% de periodontite. Por outro lado, os achados do presente estudo são similares aos relatados em outro estudo brasileiro feito com diabéticos tipo l e de faixa etária semelhante, no qual também foi encontrada alta prevalência de periodontite $(9,8 \%)(28)$.

Embora a gengivite ocorra de forma quase universal, apenas $10 \%$ da população adulta desenvolve DP na sua forma mais grave (29). A prevalência de gengivite na população estudada foi de $20,8 \%$. Índices ainda mais elevados de sangramento gengival foram relatados em adolescentes franceses com DMI (48\%) quando comparados aos controles (26\%) (30) e em outros estudos (17), mas esse achado não é consensual já que prevalência de $8,8 \%$ de gengivite também foi descrita em diabéticos tipo 1 de faixa etária semelhante à da população estudada (28).

A ocorrência de gengivite está associada, especialmente, a maus hábitos de higiene bucal. Aproximadamente $60 \%$ dos pacientes examinados relataram escovar os dentes três ou mais vezes ao dia e quase $70 \%$ disseram não possuir hábito do uso diário do fio dental. Não foram encontradas associações significativas entre esses dois cuidados e o percentual de sítios sangrantes. Por outro lado, esse percentual esteve significativamente associado ao IPL e, consequentemente, à higiene bucal. O percentual de pacientes com gengivite foi significativamente maior no grupo com placa abundante. A associação da gengivite apenas com IPL, dado de avaliação objetiva, pode significar, portanto, que a informação dos pacientes sobre hábitos de higiene não tenha sido suficientemente precisa. Corroborando essa hipótese, a maior ocorrência de gengivite no sexo masculino está de acordo com maior IPL encontrado nas crianças e adolescentes desse gênero.

O DM é considerado um dos principais fatores de risco sistêmico para desenvolvimento da DP e tanto a duração do diabetes quanto o grau de controle glicêmico podem influenciar o seu aparecimento e evolução (3-5).

Estudos observacionais conduzidos em crianças diabéticas durante 5 a 10 anos mostraram perda de inserção periodontal e aumento de SS durante o período, em maior proporção que nos grupos controle, com significativa associação entre o tempo de DM e a doença periodontal $(23,24,31)$.

A avaliação dos 168 indivíduos, no presente trabalho, também mostrou evidências de associação entre os parâmetros periodontais e o tempo de diabetes. Os resultados sugerem que pacientes com DMl de duração superior a cinco anos possuem maior risco de desenvolver DP, como já vem sendo descrito $(19,24,28,31)$. É também possível que o maior percentual de alterações periodontais em crianças e adolescentes com dentição permanente esteja relacionado ao maior tempo de DM, dado não explorado devido ao pequeno número de eventos. Outra possibilidade é que esses pacientes apresentassem pior controle glicêmico.

O mau controle metabólico poderia colaborar no estabelecimento da DP devido aos defeitos na função neutrofílica, alterações na renovação do colágeno e no padrão normal de cicatrização, além de alterações vasculares, entre outras $(3,9,10,22)$. O grau de controle metabólico tem sido considerado o maior fator de risco associado com a extensão e a gravidade da DP.

Observou-se que pacientes com mau controle glicêmico tiveram maiores percentuais de sítios afetados na PS e maior perda de inserção clínica. Dos pacientes com gengivite, $80 \%$ apresentavam mau controle. Temse sugerido que o grau de controle metabólico pode estar relacionado à destruição periodontal acelerada, independentemente do avanço de idade dos pacientes diabéticos $(19,32,33)$.

Deve ser considerado, também, que assim como o estado diabético pode contribuir para alterar os padrões de evolução da DP sendo considerado fator de risco, a DP pode contribuir para o mau controle glicêmico. Infecções periodontais podem predispor o paciente à resistência à insulina, desencadeando um estado de hiperglicemia crônica, além de dificultar o controle da glicemia do paciente $(8,13,16)$.

Os achados do presente estudo sugerem, portanto, que DM de maior duração e controle metabólico ina- 
dequado podem ser considerados variáveis preditoras de risco para a suscetibilidade à DP. É provável que a melhora no controle metabólico de pacientes diabéticos possa contribuir para minimizar os danos aos tecidos periodontais. Ao lado das medidas de prevenção e rastreamento para diagnóstico precoce das complicações crônicas já reconhecidas e amplamente adotadas para os pacientes diabéticos, esse estudo sugere que é necessário valorizar também a avaliação oral.

Dessa forma, ressalta-se a importância do exame clínico e do diagnóstico precoce das condições periodontais em indivíduos diabéticos jovens, além da conscientização das equipes de orientação para um atendimento que possibilite a implementação de adequadas estratégias de prevenção e intervenção anteriores à ocorrência de possíveis danos aos tecidos periodontais.

Agradecimentos: este trabalho teve incentivo do Conselho Nacional de Desenvolvimento Científico e Tecnológico $(\mathrm{CNPq})$ por meio de concessão de bolsa de mestrado à pesquisadora Andréa Cristina Vilan Xavier.

Declaração: os autores declaram não haver conflitos de interesse científico neste artigo.

\section{REFERÊNCIAS}

1. Beck JD, Offenbacher S. Oral health and systemic disease: periodontitis and cardiovascular disease. J Dent Educ. 1998;62(10):859-70.

2. AAP - American Academy of Periodontology. Parameters of care. J Periodontol. 2000;71:847-83.

3. Grossi SG, Genco RJ. Periodontal Disease and DM: A two-way relationship. Ann Periodontol. 1998;3:51-61.

4. Oliver RC, Tervonen T. Diabetes. A risk factor for periodontitis in adults? J Periodontol. 1994;65(5 Suppl):530-8.

5. Oliver RC, Tervonen T. Periodontitis and tooth loss: comparing diabetics and general population. J Am Dental Assoc. 1993;124(12):71-6.

6. Organização Mundial da Saúde. Levantamento epidemiológico básico de saúde bucal: manual de instruções. 3 Ed. Genebra: OMS; 1991.

7. Sociedade Brasileira de Diabetes (SBD). Estatísticas do Diabetes no Brasil e no mundo. Available from: http://diabetes.org.br/imprensa/estatisticas/index.php (acessado em 27/nov/2008).

8. Albandar IM. Global risk factors and risk indicators for periodontal diseases. Periodontology 2000. 2002;29:177-206.

9. American Diabetes Association. Standards of medical care in diabetes. Diabetes Care. 2008;31 Suppl 1:S12-54.

10. Taylor GW. Bi-directional interrelationships between diabetes and periodontal diseases: an epidemiological prospective. Ann Periodontol. 2001;6:99-112.

11. Alves C, Andion J, Brandão M, Menezes R. Mecanismos patogênicos da doença periodontal associada ao diabetes melito. Arq Bras Endocrinol Metab. 2007;51(7):1050-7.

12. Quigley G, Hein $\mathrm{H}$. Comparative cleansing efficiency of manual and power brushing. J Am Dent Assoc. 1962;65:26-9.
13. Ainamo J, Bay I. Problems and proposals for recording gingivitis and plaque. Int Den J. 1975;25(4):229-35.

14. López NJ, Smith PC, Gutierrez J. Periodontal therapy may reduce the risk of preterm low birth weight in women with periodontal disease: A randomized controlled trial. J Periodontol. 2002;73(8):911-24.

15. López NJ, Da Silva I, Ipinza J, Guitérrez J. Periodontal therapy reduces the rate of preterm low birth weight in women with pregnancy-associated gingivitis. J Periodontol. 2005;76(11 Suppl):2144-53.

16. Chase HP, Jackson WE, Hoops SL, Cockerham RS, Archer PG, O'Brien D. Glucose control and the renal and retinal complications of insulin-dependent diabetes. JAMA. 1989;261(8):1155-60.

17. Lalla E, Cheng B, Sal S, Tuches S, Greenberg E, Goland R, et al. Periodontal changes in children and adolescents with diabetes: a case control study. Diabetes Care. 2006;29(2):295-9.

18. Lalla E, Kaplan S, Chang SJ, Roth GA, Celenti R, Hinckley K, et al. Periodontal infection profiles in type 1 diabetes. J Clin Periodontol. 2006;33(12):855-62.

19. Lalla E, Cheng B, Sal S, Tucher S, Greenberg E, Goland R, et al. DM promotes periodontal destruction in children. J Clin Periodontol. 2007;34:294-8.

20. Lalla E, Cheng B, Sal S, Tucher S, Greenberg E, Goland R, et al. Diabetes-related parameters and periodontal conditions in children. J Periodontol Res. 2007;42(4):345-9.

21. Löe H. Periodontal disease: the sixth complication of Diabetes Mellitus. Diabetes Care. 1993;16(1):329-34.

22. Mealey B, Ocampo G. Diabetes Mellitus and periodontal diseases. Periodontology 2000. 2007;44:127-53.

23. Novaes Jr AB, Pereira AL, Moraes DE, Novaes AB. Manifestations of insulin-dependent DM in the periodontium of young Brazilian patients. J Periodontol. 1991;62:116-22.

24. Novaes Jr AB, Silva MAP, Batista Jr EL, Anjos BA, Novaes AB, Pereira ALA. Manifestations of insulin-dependent DM in the periodontium of young Brazilian patients. A 10 -year follow-up study. J Periodontol. 1997;68:328-34.

25. Costa FO, Cota LOM, Costa JE, Pordeus IA. Periodontal disease progression among young subjects with no preventive dental care: A 52-month follow-up study. J Periodontol. 2007;78(2):198-203.

26. Albandar IM. Global Epidemiology of periodontal diseases in children and young persons. Periodontology 2000. 2002;29:153-76.

27. Collins J, Carpio AM, Bobadilla M, Reyes R, Gúzman I, Martínez B, et al. Prevalence of clinical attachment loss in adolescents in Santo Domingo, Dominican Republic. J Periodontol. 2005;76:1450-4.

28. Cianola LJ, Park BH, Burk E, Mosovich L, Genco RJ. Prevalence of periodontal disease in insulin-dependent DM (juvenile diabetes). J Am Dent Assoc. 1982;104:653-60.

29. Stamm JW. Epidemiology of gingivitis. J Clin Periodontol. 1986,13(5):360-70.

30. De Pommereau V, Dargent-Paré C, Robert JJ, Brion M. Periodontal status in insulin-dependent diabetic adolescents. J Clin Periodontol. 1992;19(9 Pt 1):628-32.

31. Firatli E. The relationship between clinical periodontal status and insulin-dependent DM. Results after 5 years. J Periodontol. 1997;68:136-40.

32. Westfelt E, Rylander H, Blohme G, Joanasson P, Lindhe J. The effect of periodontal therapy in diabetes. J Clin Periodontol. 1996;23:92-100.

33. Lim LP, Tay FBK, Sum CF, Thai AC. Relationship between markers of metabolic control and inflammation on severity of periodontal disease in patients with DM. J Clin Periodontol. 2007;34:118-23. 